\title{
Revealing Hidden Local Cultural Heritage through a Serious Escape Game in Outdoor Settings
}

\author{
Stavroula Tzima ${ }^{1,2}$, Georgios Styliaras ${ }^{3, *(1)}$ and Athanasios Bassounas ${ }^{4}$ \\ 1 Department of Fine Arts and Arts Sciences of Arts, University of Ioannina, 45110 Ioannina, Greece; \\ stzima@uoi.gr or sjima@upatras.gr \\ 2 Department of History and Archaeology, University of Patras, 30100 Agrinio, Greece \\ 3 Department of Food Science \& Technology, University of Patras, 30100 Agrinio, Greece \\ 4 Department of Early Childhood Education, University of Ioannina, 45110 Ioannina, Greece; ppr00138@uoi.gr \\ * Correspondence: gstyl@upatras.gr
}

Citation: Tzima, S.; Styliaras, G.; Bassounas, A. Revealing Hidden Local Cultural Heritage through a Serious Escape Game in Outdoor Settings. Information 2021, 12, 10. https://dx.doi.org/10.3390/ info12010010

Received: 27 November 2020 Accepted: 22 December 2020 Published: 25 December 2020

Publisher's Note: MDPI stays neutral with regard to jurisdictional claims in published maps and institutional affiliations.

Copyright: () 2020 by the authors. Licensee MDPI, Basel, Switzerland. This article is an open access article distributed under the terms and conditions of the Creative Commons Attribution (CC BY) license (https: / / creativecommons.org/ licenses/by/4.0/).

\begin{abstract}
Escape Rooms are presently considered a very popular social entertainment activity, with increasing popularity in education field, since they are considered capable of stimulating the interest of players/students and enhancing learning. The combined game mechanics have led to blended forms of Escape Rooms, the Serious Escape Games (SEGs) and the hybrid type of Escape Rooms that uses Augmented Reality (AR)/Virtual Reality technology, a type that is expected to be widely used in the future. In the current study, the MillSecret is presented, a multi-player Serious Escape Game about local cultural heritage, where the players must solve a riddle about the cultural asset of watermills. MillSecret uses AR technology and it was designed to be conducted in the real-physical environment and in an informal educational context. The paper describes the game, its implementation, the playing process, and its evaluation, which aimed to study the feasibility of game conduction in outdoor settings and the views and experience of players with the game, the local cultural heritage and local history. Evaluation results reveal, among other findings, a very positive first feedback from players that allows us to further evolve the development of the game.
\end{abstract}

Keywords: cultural heritage; games; Augmented Reality

\section{Introduction}

In recent years, gaming, game-like applications, digital games and Serious Games (SGs) [1], and lately Escape Rooms have gained interest and are used both in the educational field $[2,3]$ to meet teaching needs on various topics, such as history and cultural topics, chemistry, astronomy etc. and in the cultural heritage field [4]. In education, they are combined with game-based teaching methods and used in formal, non-formal and informal learning contexts for diverse purposes, such as socialization and teaching improvement [2,5]. In cultural heritage, gaming and game-like applications offer the opportunity to re-live and re-interpret the past, provide attractive experiences to visitors and especially to the younger generations by using modern digital technologies, offer a novel way to museums and institutions to communicate with public and contribute to the cultural heritage preservation and dissemination [1,6-8]. Escape Rooms are presently considered a very popular social entertainment activity, with increasing popularity in education field, since they are considered capable of stimulating the interest of players/students and enhancing learning [2-4]. The combined game mechanics have led to blended forms of Escape Rooms, the Serious Escape Games (SEGs) with a use of many prospects [9] and the hybrid type of Escape Rooms that uses Augmented Reality (AR)/Virtual Reality (VR) technology, a type that is expected to be widely used in the future $[3,10]$.

In the current study, a Serious Escape Game, the MillSecret, for local heritage and history will be presented in order to cover the need to engage young generations with cultural heritage and history in an attractive way [7]. The game uses key concepts of 
Escape Rooms which are considered "a competitive market" [4] (p. 9) and "ideal for places of informal learning that emphasize a physical location" [4] (p. 25). Millsecret uses AR technology and it was designed to be conducted in the real-physical environment and in an informal educational context where more research is needed [4]. The evaluation of the game aimed to study the feasibility of game conduction in outdoor settings, the acceptance of if this type of game players/visitors and the views and experience of players with the game and the local cultural heritage.

\section{A Brief Field Review}

\subsection{Cultural Heritage and Games}

In the cultural heritage field and especially for museums, games are considered an effective tool to motivate visitors and provide engaging and memorable user experiences [11,12]. Various applications and approaches have been developed in order to communicate and safeguard cultural heritage [13] and to study fundamental concepts and features such as presence, immersion [14], visitors profiling and museum advertising [11]. These applications use different technologies such as livestreaming [13], 3D environments [14] and geo-location [15] in various ways (e.g., virtual exhibitions, mobile apps). AR and VR can enhance the dissemination and appreciation of cultural heritage [15] through engaging and immersive experiences such as serious games [16]. In the case of AR games, they must be adapted to the physical world [17]. They are developed with diverse purposes such as "to engage visitors with the architectural contents of the site" in an AR construction game [18] (p. 22) or to "bring back to life" archaeological sites that have been lost" in case of a marker-based AR mobile app that was developed about the Koguryo tombs with painted tumuli and a fragile and difficult accessed indoor environment [19] (p. 2).

According to Koutroumanos and Labropoulos, several studies focus on the use of AR games about the local history and civilization. These games take place in the natural environment, require exploration, and use of mobile devices, promote physical activity, teamwork, and collaboration, and create a positive attitude for similar applications [20]. In their research, they implemented an adventure game, the game "Salamis", where players had to explore real sites-monuments, use the AR application, collect information. and solve riddles [20]. Di Paola, Inzerillo and Alognaa designed a cultural heritage video game, which they classified more as an Action-Adventure Game and Entertainment Game than a Serious Game. In their game, AR was embedded into the VR environment with a core role by providing the keys for the next levels [17]. Shin, Kim, and Woo present the design of a mobile AR game for the site of Daereungwon, based on Points of Interest (PoIs) features, visual marks, and GPS to provide an on-site experience. According to their research, other studies which apply gamification and AR in cultural heritage field mainly focus on location-based games [21]. Haahr [22] studied game experience and more specifically location-based AR games for cultural heritage sites that take place in the actual site and retain the sense of presence in the real environment. According to Haahr, the term presence in game experiences that take place in a real cultural heritage site cannot be defined and studied as in virtual environments in the sense of "telepresence", but more generally as a mediated experience, close to the natural way of perceiving the real environment. Regarding the "immersion" term, they study the types of sensory, challengebased and imaginative immersion. They emphasize the need for a connection and balance between the physical world and the game world through the interaction of the elements of the two worlds, the creation of audiovisual material and game mechanics that will not emphasize only the game world and will not distract player's attention from the physical environment [22]. Nofal et al. [23] present TouchTomb, "a tangible gamified installation" for students aged 10 to 14, who visit Neferirtenef tomb-chapel in the context of school visits. They built a physical, realistic wall in the size of the true part of the inner west wall of the chapel to work as the interactive tangible interface. The installation was deliberately placed just outside the original chapel and hosted three game categories to communicate 
respectively different types of heritage information (architectural, historical, and artistic). Game setup included a 3D puzzle, cards, LED lights and "a LED strip connected to an Arduino Mega" to indicate progress and valid placements and aimed to study how the installation affects the interaction with the tangible and real interface [23] (pp. 6-9).

\subsection{Serious Games}

Serious Games are described as "games with educational purposes" [24] (p. 1). The learning goals are achieved "through a fun experience" where various factors (usability, competition mechanism etc.) determine players engagement [24] (p. 1). Therefore, the primary goal of Serious Games is not entertainment but learning and the game-based environment is used as the medium that will convey the learning content $[7,25,26]$. Serious games, "also referred as educational games" [16] (p. 4), have many common features with all types of games (game mechanisms, rules, audiovisual experience, element of challenge etc.) [7], they can engage players in the experience, stimulate and retain their interest in learning and encourage perseverance in completing tasks [16,25]. Serious games are used in various fields such as cultural heritage, education, healthcare [27] and are preferred by students more than traditional methods [28]. They are considered that can be effective tools [17] for learning and training $[17,18]$ but are less attractive to young generations than Action and Adventure Games [17]. Serious Games mostly focus on educational outcome and less on entertainment and their transformation to Action and Adventure Games is proposed as a challenge [17]. Yallihep and Kutlu [28] concluded that mobile serious games can effectively enhance students' achievements, whereas further studies are required to investigate the role of mobile serious games as educational tools [28].

\section{Serious Games in Cultural Heritage}

SGs can effectively communicate cultural heritage by experiences that combine learning and entertainment, trigger interest, motivate further engagement with cultural heritage [29] and help visitors gain experience and knowledge [12,30]. They place emphasis on historical reconstruction, on cultural and heritage awareness, on history education, on museum visits, on Intangible Cultural Heritage (ICH) safeguarding and transmission [6,8]. They have been used in various forms such as action, strategy, and simulation games, in formal and informal educational context (e.g., home, school, city) [24]. SGs aim to "increase user engagement, attract new visitors and provide cultural content in a way that can lead to long-lasting experience" [27] (p. 1). SGs, AR and Digital Storytelling are among the digital technologies used and combined in cultural applications [31]. Motivation and user engagement are considered key factors in a cultural heritage learning experience and serious games and game mechanics can effectively raise learners' interest, trigger their motivation, and encourage them to participate in situated learning experiences [25].

In cultural heritage activities, emphasis is given to boost user's participation in shaping their cultural experience. User's cultural profile and background is considered a key factor that positively affects user's experience along with "user expectation, motivation and feelings" [31] (p. 3). Additionally, the simple features and function of a cultural product contribute to a positive user experience [31]. Finally, SGs are different from games in other fields: Their purpose and objectives differ [12] and the cultural context differs compared to other fields since the content in cultural sites is complex, visitors are diverse and usually they visit a place only once with limited available time [11].

\subsection{Escape Rooms}

Escape Rooms are team-based activities, usually of five players or more with a time limit of 15-120 min in most games, which can take place in various places e.g., in a meeting room turned into a playing stage [2], or a classroom [3]. They include diverse challenges [10] that require multiple skills and abilities such as team spirit, cooperation, task management, emphasis on detail etc. [4]. According to Fotaris and Mastoras [3], in most Escape Rooms, the objects that contain clues are only physical, such as locks, while the mix of physical and 
digital objects, such as QR codes, are less used, but the hybrid type of Escape Rooms that use AR/VR technology is expected to be widely used in the future [3]. In addition, Escape Rooms are considered "a competitive market" [4] (p. 9) and therefore they constitute a field of multiple future applications and research.

Nicholson defines Escape Rooms as "live-action, team-based games where players discover clues, solve puzzles, and accomplish tasks in one or more room in order to accomplish a specific goal (usually escaping from the room)" [4] (p. 1). Escape Rooms probably appeared in Japan in 2007, maybe even earlier [10] and expanded afterwards in other countries $[2-4,10]$. According to Nicholson the $65 \%$ of Escape Rooms owners were inspired by other Escape Rooms and the rest 35\% by other sources such as adventure movies. The precursors of Escape Rooms were the live-action role-playing games, pointand-click adventure games and escape-the-room digital games, puzzle hunts and treasure hunts, interactive theater and haunted houses, adventure game shows and movies and the themed entertainment industry [4].

In the field of education, according to Fotaris and Mastoras [3], Escape Rooms "are based on a social-constructivist approach (Vygotsky, 1978)", where players learn through a real-time experience and various challenges such as solving puzzles. The theme also varies since player's escape is not the only theme, but other themes as saving a fictional character [3] are also used. They aim to meet teaching needs on various topics, such as history and cultural topics, chemistry, astronomy, etc. [4] by providing an attractive learning context for students and an effective teaching tool for teachers [9]. They are considered to be an innovative approach that offers multiple benefits and can effectively enhance learning through a real, active, collaborative, and engaging experience. More specifically, they promote communication and collaborative teamwork [4], activate interest, motivation, and willingness to learn [2] and they enhance social and analytical skills such as social interaction, critical thinking etc. [3]. According to Nicholson, "being part of a spectacle, feeling heroic, and engaging with something challenging" are the required conditions that will immerse players in the game [4] (p. 26).

\subsection{Serious Escape Games}

The authors in [9] define "Escape Game (or Escape Room), as a multi-player life-size escape game, which typically involves escaping from a room or a succession of rooms in a limited time" where players have to find clues and objects to solve riddles [9] (p. 1). They proposed a type of Escape Games for teaching, the Serious Escape Games (SEG), the adapted form of a serious game, as well as a model, the Serious Escape Game Model (SEGAM) and methods to design these type of games [9]. In SEGs they combined and adapted learning, Serious Games, DISC (Domain, Interaction, Scenario, Context) model and ATMSG (Activity Theory-based Model for Serious Games) elements [9].

In the current study, a Serious Escape Game for local heritage and history is presented. The game was developed based on escapED framework [32] and SEGAM model [9] and relevant guidelines of other studies, as presented throughout Section 3. It is designed to be conducted in the real-physical environment, which can be linked with the virtual through games [24]. It has been used as the 3D environment of AR applications augmented with digital content (text, images, 3D objects, etc.) [25] and therefore can constitute a meaningful environment.

\section{Game Description and Implementation}

\subsection{The Game}

MillSecret is a multi-player Serious Escape Game of one level. Players must detect hidden physical objects and hidden digital information using the AR app in order to solve a riddle about the cultural asset of watermills. The game was designed and developed to be played by players of all ages, at least 10 years old (visitors, residents, students), with no special knowledge or experience on Escape Room games. It was designed to have a short duration in order to avoid player fatigue, to be played by 3 to 6 players, based on 
cooperation without a predefined time limit. It was deliberately chosen not to impose a time limit, in line with other studies [10], in order to have more time to study the dynamics and cohesion of the teams, the reactions and attitude of the players, both regarding the game (e.g., degree of difficulty, acceptance) and the local history and local cultural heritage.

\subsection{Objectives and Learning Process}

The game is designed to be an informal learning experience based on the educational strategies of active and experiential learning aiming to knowledge acquisition, behavioral change, and problem-solving skills development. Knowledge acquisition is expected to be achieved through the solution of the riddle that requires information about the watermill and the history of the place as a prerequisite to solve the math quiz, which consists of simple algebraic problems that include information acquired through the AR app (e.g., year of the landslide that destroyed the monuments). Problem solutions are clues that lead to the riddle solution. These dependences intend to make players watch the history of the place carefully in order to get the required data, to use their knowledge in mathematics in a creative and enjoyable way and thus to strength their self-confidence and motivate further learning. Players will need to put emphasis on this information in order to combine it with the rest of the data to find the solution and get a reward. In this way, the AR app supports the learning objectives of the game. Thus, at the end of the game, the participants are expected to have acquired knowledge and to show increased interest for the cultural asset of watermills, the local watermill and the history of the place and its value. Consequently, the goal of behavioral change in terms of interest in local tradition and history is expected to be achieved. The game is designed to be played by a group of players and thus competition and collaboration are the core mechanisms to boost players' motivation and the successful completion of the game. Additionally, special skills are not required, and the game can be played by teams of players with different levels of experience, knowledge, and skills, which are considered to be the most successful teams [9]. Finally, the problem-solving challenge, the solution of the riddle, is expected to create a more interesting, creative, and fun experience.

\subsection{The Hybrid Reality Environment}

The game was designed to be implemented at the physical environment, it can be played only in the selected place and is structured as a single scene, the mix of the real environment with the digital (Figure 1). The place was selected due to the historicity of the area (Figure 1a). In that place, from the Byzantine period, there was a women's Christian Orthodox monastery, which was later evolved into a church. There were also watermills / flourmills with fulling mills which were destroyed by a major landslide [33]. Now, there are the ruins of the old church (Figure 1b) and a watermill (Figure 1d) that was built immediately after the landslide and operated until the previous decade. A field research and extensive study of historical data of the area, we carried out during recent years, provided historically accurate and realistic data that supplied the digital content of the AR app (Figure 1c,e) [34-37]. The use of AR technology complements the world, connects the present with the past and the physical objects with the digital ones and along with competition, challenge, exploration of the environment and the rest game elements, leads to user's immersion and engagement into a compelling experience of local culture and history, according to the studies presented in $[9,25]$. 


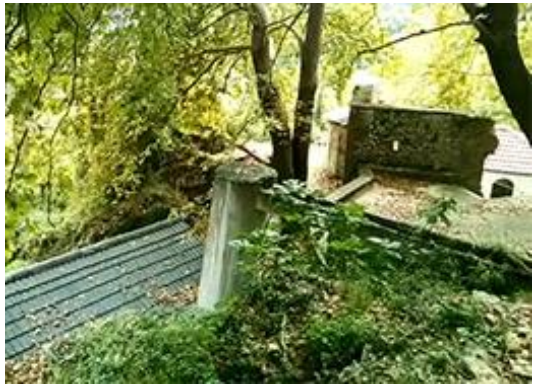

(a)

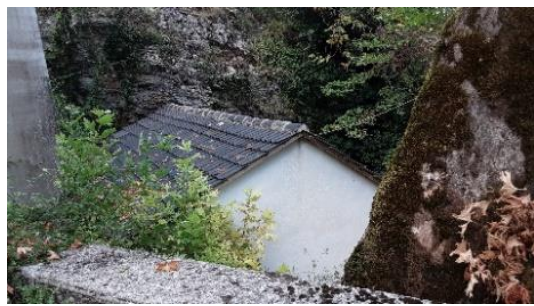

(d)

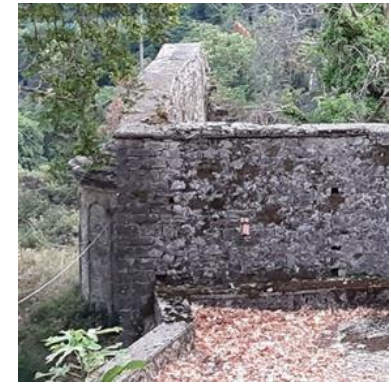

(b)

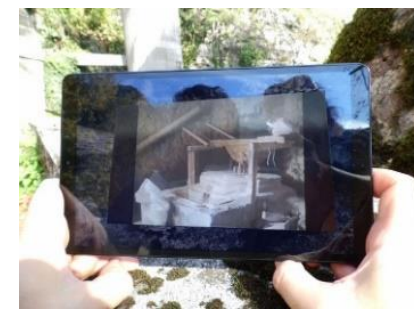

(e)

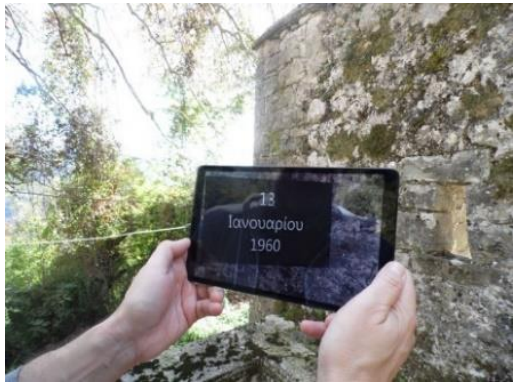

(c)

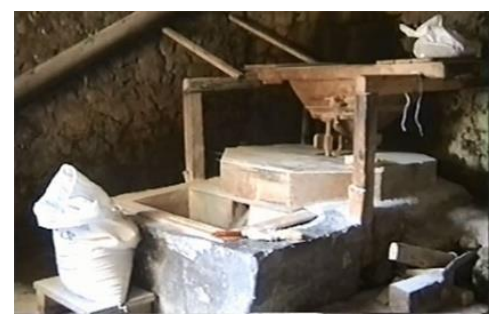

(f)

Figure 1. The hybrid reality environment. (a) The physical environment; (b) The ruins of the old church; (c) The old church's building triggers the AR app and reveals its history; (d) Outside the watermill; (e) The watermill triggers the AR app and reveals its history; (f) Inside the watermill in the operational days of the previous decades.

\subsection{Theme}

The game's plot is built around the fiction that the mill craftsman has hidden clues in various places in the area outside the watermill, in order to protect his secret about the way the millstone is constructed. Players must solve a riddle to learn the secret. The solution will come from the right combination of hidden clues and as a reward for solving the puzzle, the video of the representation of the local myth is played by the AR application.

\subsection{Structure and Implementation}

The game environment is built in a hybrid organization [4] (Figure 2), combining physical and digital objects with the real environment of the selected local site in order to provide an immersive atmosphere.

The physical objects used are everyday objects (wooden box, padlock and key, fabric bag) and objects constructed for the needs of the game (Figure 3).

The digital objects present historical data about site's monuments (Figure 4a,b) and a local myth (Figure 4c), include photos, narration, and video, all embedded in the imagebased AR app. Processing of the digital material was performed using Adobe Suite and Windows Movie Maker. The AR app was developed using Unity 3D and Easy AR platform and installed on a tablet that was provided to players. To ensure image recognition, more than one images of the monuments were used to activate the AR app and specific positions were marked to help players in case of difficult light conditions. 


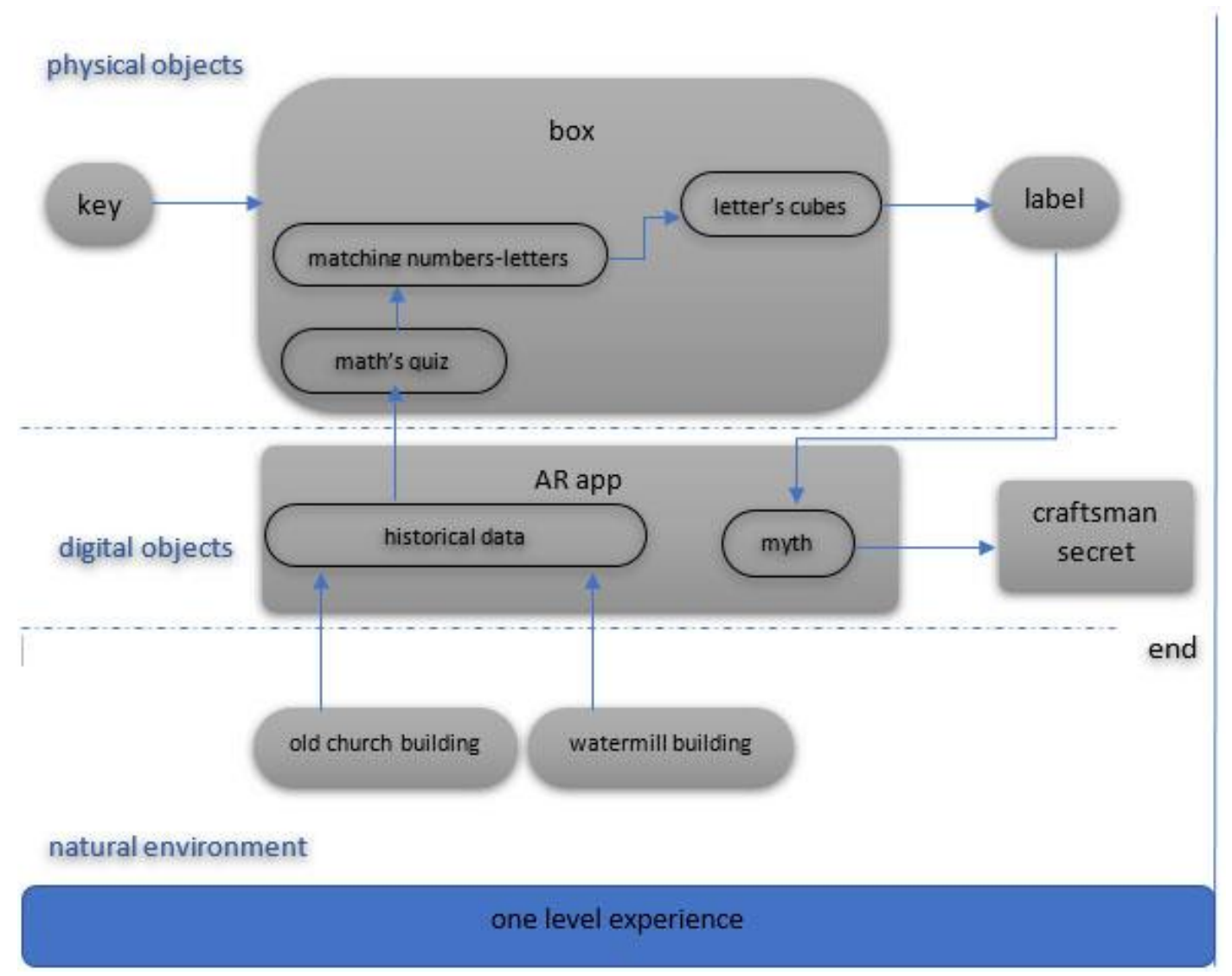

Figure 2. The hybrid organization of the game.

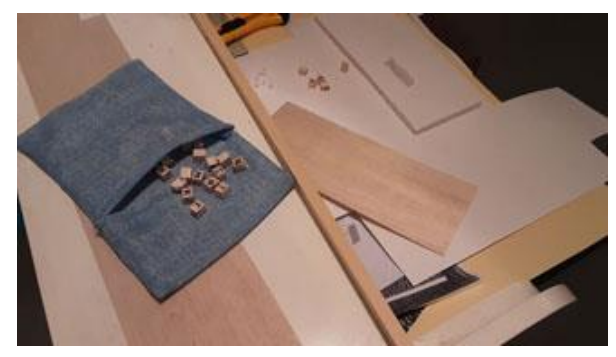

(a)

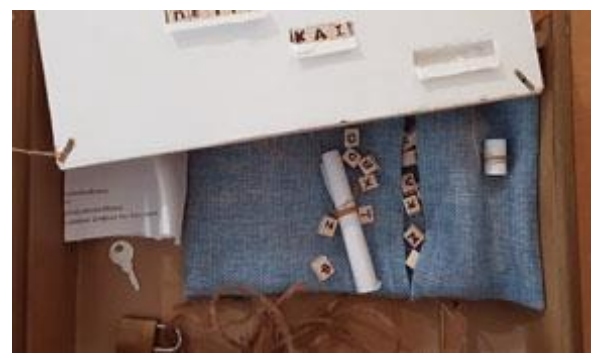

(b)

Figure 3. (a) Constructing the foam board label and the wooden cubes with letters; (b) The key, the box, the math's quiz, clues for solving the quiz, a bag with letters and the back side of the label where players had to put the missing word.

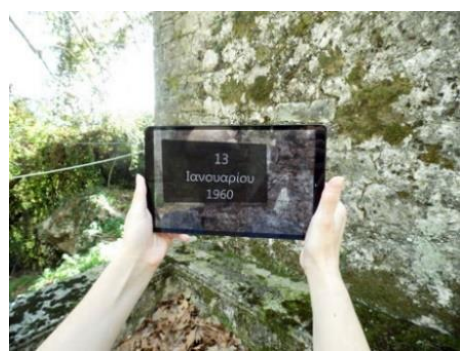

(a)

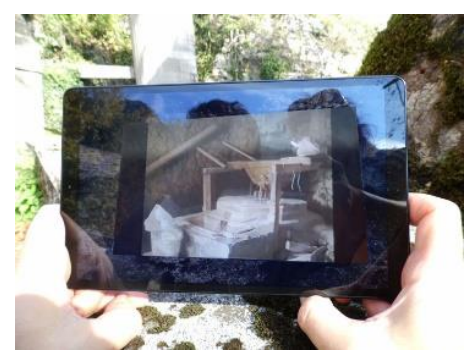

(b)

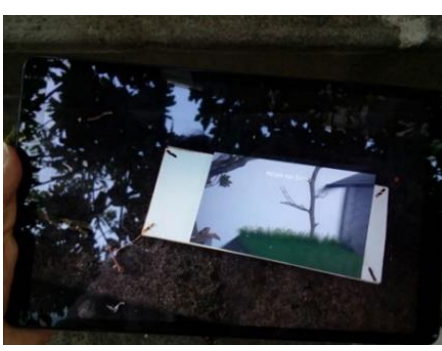

(c)

Figure 4. The digital objects provided through AR app's content. (a) Historical information about the old church includes the hidden digital clue; (b) The watermill in the operational days of the past (c) The local myth representation, displayed at the end of the game, reveals the craftsman's secret. 


\subsection{Objects, Clues and Rewards}

The physical objects that players must search for are the key, the box, and the label, which were hidden in purposefully selected spots (Figure 5). More specifically, the label was hidden at a spot that could be easily found by players in order to encourage them, as it is proposed in [2], the box was carefully hidden in a more difficult spot because it was an important object that contained other clues and objects, while the key was placed on an obvious spot, but carefully hidden in order to make the game more challenging. The AR app contains a hidden digital clue about the monuments of the site, the old church building (Figure 4a) and the watermill's building (Figure $4 \mathrm{~b}$ ) and it is activated by these monuments. Also, the AR app contains the craftsman's secret that will be displayed as a reward to players, activated by the riddle's solution at the end of the game (Figure 4c).

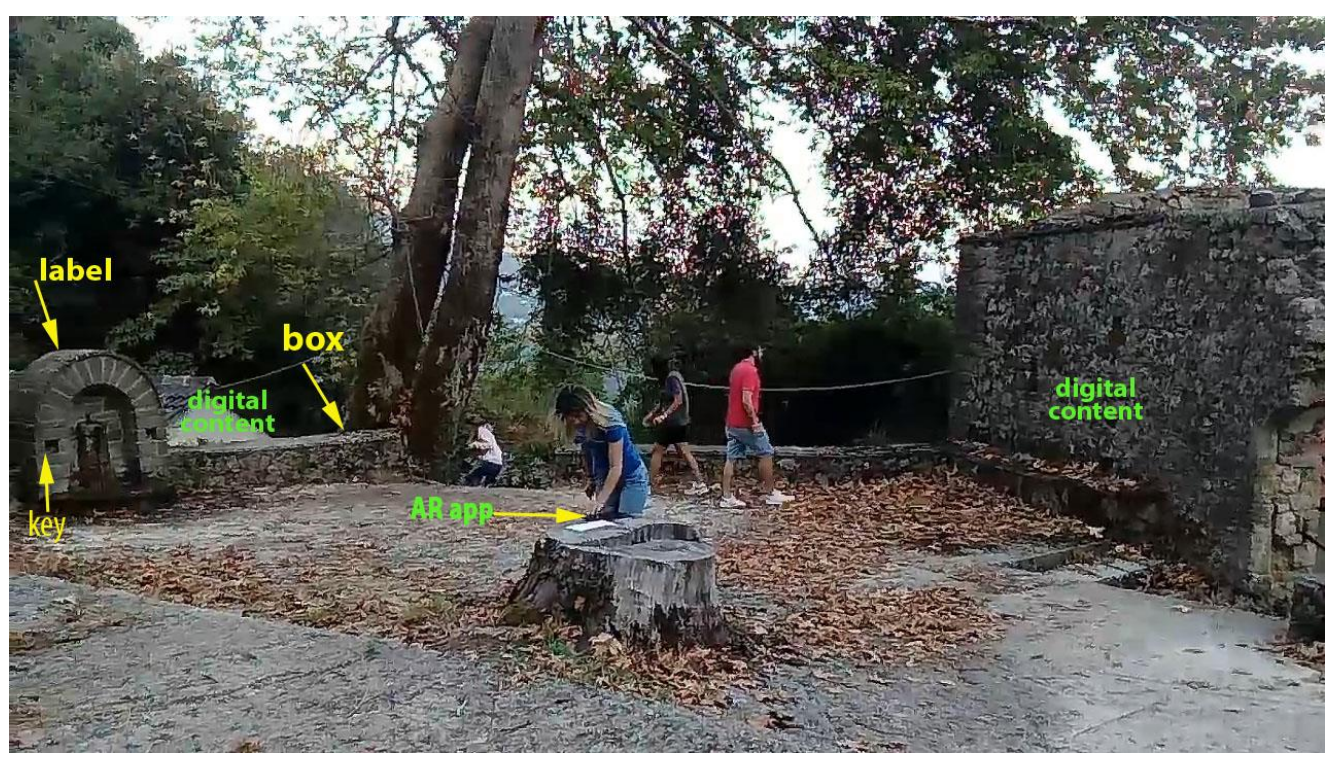

Figure 5. Location of the hidden objects.

Red herrings (misleading clues and objects) were not used because in the physical environment, a variety of objects exist that could mislead players and therefore it would increase the difficulty level. According to Nicholson, $50 \%$ of escape room designers do not include red herrings [4]. Also, extra clues were not used since the instructor would provide hints in case players needed or asked for (e.g., get stuck) [2] and therefore the standard mode of playing the game was followed [4].

\subsection{Objects' Role and Players' Actions}

The game is based on a narrative design in order to keep player's interest, is a single experience with a non-linear flow, a fact that allows each player to take his own area of responsibility and contribute to the quick solving of the puzzle. Players have to observe, focus, think and cooperate in order to solve the riddle. Initially, they can work independently to find the three physical objects and to use the AR app and then they must cooperate in order to combine clues and find the solution. The role and usefulness of the objects varied: obvious in case of the key to unlock the box and more complex with other objects. The objects will help players figure out what other objects they need to find. For example, if they find the locked box first, they will realize that they have to look for the key and vice versa. The box (Figure 3) contains a small bag with letters, a clue that explains the matching between numbers and letters, and a math's quiz where the numeric results correspond to the riddles letters and constitute the medium "to transpose the learning contents into riddles" [9] (p. 2). The math's quiz contains equations that required knowledge of elementary-high school level mathematics and data about local history contained in the AR app. The label has a carved recess at the back, which fits exactly 
the letters of the word puzzle and when players place the letters in, the AR app is activated, to display the local myth (Figure 4c).

\section{Testing and Evaluation}

\subsection{Research Questions, Methodology and Tools}

Game evaluation is based on qualitative research. The emergent sampling was deployed [38] in order to give the opportunity to anyone who wants to participate. Observation and semi-structured interviews [38] were used as qualitative data collection techniques. Observation is considered a basic method and its experiential character suits the requirements of the current study [38]. The semi-structured interview was chosen since it combines flexibility and the use of a question guide [38]. More specifically, a short but comprehensive set of questions (Appendix A) was used in the current study in order to study a set of research questions without wasting visitors' time. Content analysis was used to process the data, which are organized and presented according to the research questions [39]. The research questions regarding a Serious Escape Game (MillSecret) about the local history and local cultural heritage, are:

1. What are the impacts of the Serious Escape Game on team's behavior?

2. Is it and to what extent accepted by players/visitors?

3. Can it and to what extent positively affect players' attitudes toward local cultural heritage?

\subsection{Participant's Profile}

Game play took place in 9th August 2020 with 16 players (hereinafter referred to as P1 for the 1st player etc.), six female and 10 male, of age ranging from 13 to 31 years. There were five school students, eight university students and three older players. Thirteen of the participants were regularly visiting the village, at least twice a year, spending enough time there, while the other 3 players were visiting the village for the first time. Regarding players knowledge about the history of the site, all the 13 regular visitors of the village stated that they knew about the existence of the old church but ignored any information about its past. Additionally, they stated that they were informed about the existence of the watermill and more specifically 3 out of 13 had only heard narrations by their grandparents, 5 out of 16 had visited but without remembering details, whereas 5 out of 13 had strong memories of watermill's operation. About players' previous experience with escape room games 7 out of 16 answered that they had played escape room games in the past. Regarding the previous experience with AR applications, 9 of the 16 participants stated that they had used some AR app before (P1, P3-P7, P11, P13, P14) and "Pokemon Go" was stated as an example application they had used.

\subsection{Process}

Game evaluation included the following four stages:

1. Preparation and testing. The preparation and testing stage aimed to setup the game, to ensure player's safety and the smooth operation of the AR application according to other research guidelines $[4,9,25]$. More specifically, preparation and testing lasted 5 days and special emphasis was given on the safety of the players. The play area was cleaned, leaves and broken tree branches were removed. Next, the spots where the objects would be hidden and the spots where the digital app would be activated were selected. The AR application was extensively tested in order to ensure its smooth operation, to select the most suitable and safe search area and to ensure enough space for comfortable players movements and the successful image recognition, because in the external environment the light brightness changes at different times of the day.

2. Players invitation. Players were initially informed about the purpose of the study, the game content, the game evaluation and about the playing options and then they were invited to take part in the game. More specifically, they were offered the opportunity to choose the day and time of the event and to form the teams themselves or to play 
the game as one single team. The limitations were that the number of members of each team should not exceed six people. In most games, teams have five players or more [3]; however, for the settings of the specific game (available space, number of items and quizzes), a team with more members was not considered to be an effective choice. Another limitation was the weather conditions, which should not restrict game conduction and there should be daylight for the application to be used. There was a very positive response and willingness to participate, so after the invitation there were immediate appointments for the day and time of play.

3. Game conduction (Figure 6). The game conduction stage provided a test with players in real conditions and aimed to study this type of game, the players' reactions and attitudes toward the game and the local cultural heritage and to identify possible problems, failures, etc. that were not predicted during game development. There were two game plays, with two teams competing in each play. Players were introduced to the purpose, the goal, and the rules of the game. Grouping into two teams was done by the players themselves. Players were also asked to choose whether they wanted to compete based on a time limit or on the best time. All players decided to compete for the best time. The first two teams had already decided the grouping, and both teams had the same number of players. The 3rd and 4th teams were formed just before the play. All teams decided the order of participation themselves. Before the game each team was given instructions about the purpose of the game (search for a hidden secret by solving puzzles), about the use of the application and about the area where they should search for clues. During the play, the opposing team waited its turn at another spot, without visual or acoustic contact with the game area. The players were supervised by the researcher who was present in the place the game was conducted and had the role of the game master, first of all to ensure players safety, to guide and help them in case they got stuck or disappointed by providing hints, to provide feedback and guidance in certain cases such as when looking for items outside the area or confirmation if an object was a clue for the solution of the puzzle and to ensure the smooth flow of the game, according to relevant study findings and guidelines [4,9].

4. Game evaluation. The game evaluation stage included initially a group semi-structured interview and then the debriefing-reflection phase (Figure 7). In the end of each play, a group semi-structured interview was conducted, which lasted 20-30 min and only written notes were kept. The debriefing-reflection phase is considered an important element [32] and in the current study aimed to study the views and experience of players with the game, local history, and local cultural heritage. The debriefing-reflection phase with the players lasted 15-20 min, where the researcher-instructor explained the purpose of the game, how the game was organized with the first priority being the safety of the players, what elements were used, how the documentation of the historical data was done (residents' interviews, etc.), the use of stop motion technique for the myth's representation and the AR application development. Debriefing-reflection is considered a critical component that leads to long-term learning, to better experience and to game improvement $[3,4,9]$. In the current study it was additionally used as an evaluation tool. Evaluation of learning outcomes was conducted in an indirect way, through interviews and discussion and not by a formal form like a test, which was considered inappropriate in this preliminary phase of the game. 


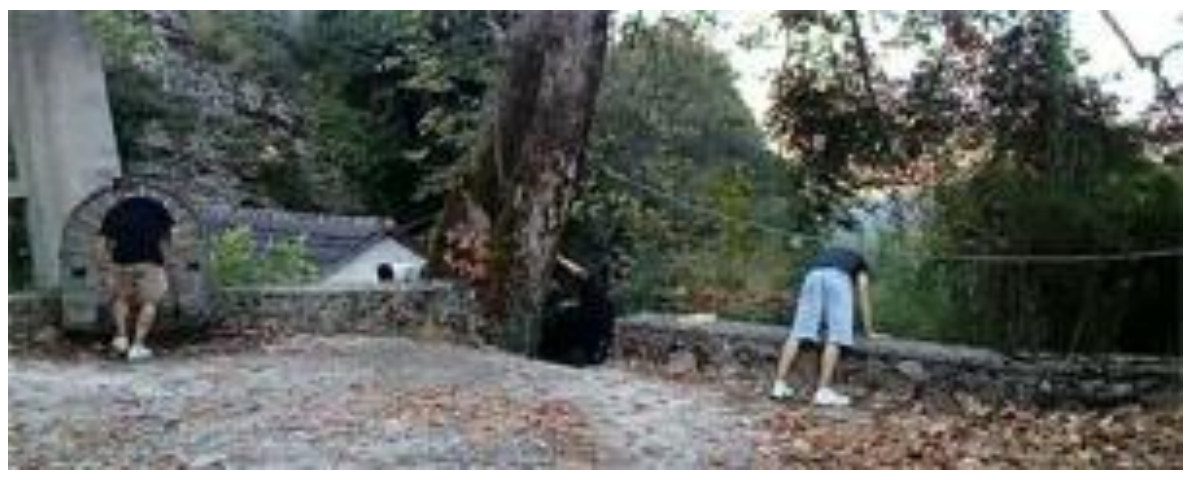

(a)

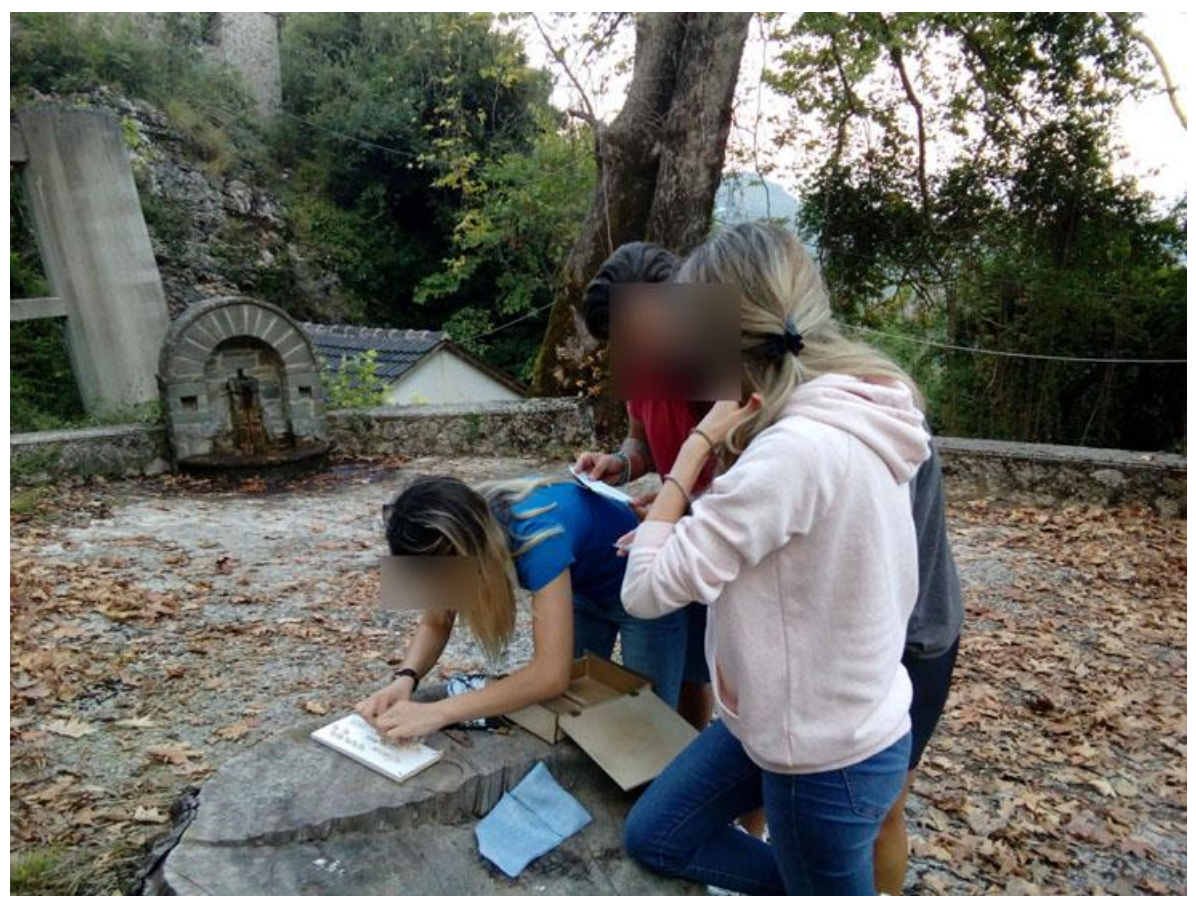

(b)

Figure 6. Game conduction. (a) Looking for hidden objects; (b) Placing letters in label's recess.

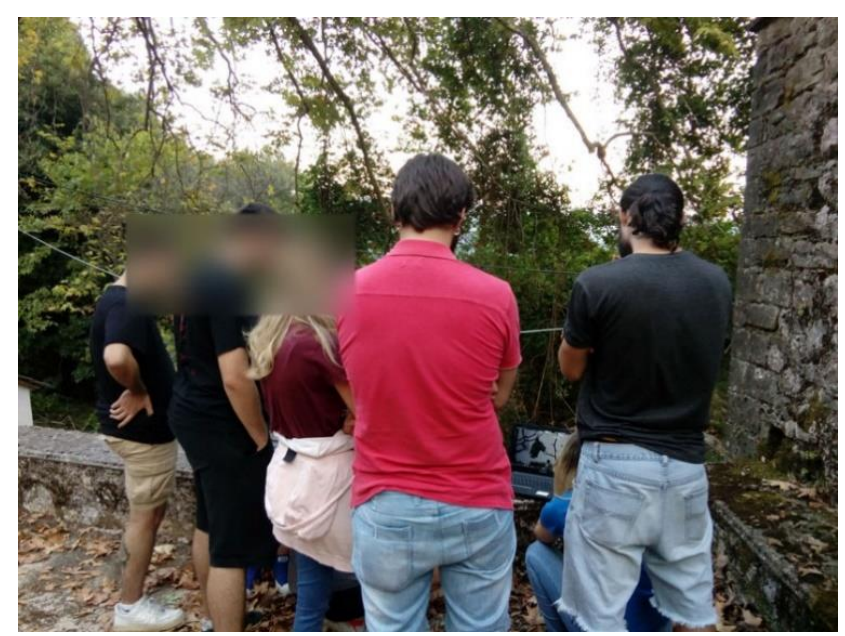

Figure 7. The debriefing-reflection phase with two teams' players. 


\section{Evaluation Results \\ 5.1. Impact on Team's Behavior/Teams' Dynamics and Cohesion}

Based on researcher's observations, the first team solved the riddle in approximately $15^{\prime}$, the second team in $27^{\prime}$, the third in $18^{\prime}$ and the fourth in $23^{\prime}$. The first team was organized and collaborated effectively (each player was looking at a different spot), they were methodical and searched in detail and without haste. The second group did not have good member coordination, they lacked intensity and rhythm. The third team was very competitive, and they played with intensity and rhythm because they wanted to beat the fourth team. The fourth team found the three items in the first five minutes but had difficulty in finding the fourth item, wasting much time. Nevertheless, they continued to play with speed, and, at that point, instructions were given to focus their search in a specific object and to search in more detail. The first and second teams played very carefully, following the game rules. The third team did not leave the field as the fourth team was playing, promising that they would just watch and not bother. In the process, however, they were joking and teasing the fourth team members, a fact that slightly annoyed and distracted the players and led to recommendations from the researcher. However, overall, the competitive spirit and the teasing between friends created a very pleasant atmosphere.

The opportunity to choose how to play the game enhanced the players' involvement and engagement with the experience. The element of friendly competition among teams activated them. As for the reward element, three players seemed surprised because they were expecting something more tangible or ordinary as reward (P2, P4, P7). The opportunity to choose the element of reward could have a more positive effect. None of the players had difficulty to use the AR app. Three groups had to watch the digital content of the AR app twice to confirm the landslide date, the key clue for the game. This fact did not cause any inconvenience to the players or any negative comments and certainly helped them remember an important event of site's history. All the players played with interest and enthusiasm and maintained this attitude until the end of the game, where they watched the video of myth's representation very carefully. All the players were careful with the space and the monuments and helped in setting up the game for the opposing team.

\subsection{Attitude Toward the Game}

To study a player's opinion about the game, they were asked four questions. The first question was "how interesting did you find the game?" along with the choices of "not at all", "a little", "moderate", "very" and "extremely", for players' convenience and time saving purposes. 8 of the 16 players described the game as "very" interesting, while the other 8 described it as "extremely" interesting. When asked the second question "what did you like about the game?", they answered: "the combination of historical elements" (P1), "teamwork" (P8), "victory" (P11), "adventure" (P9), "participation" (P13) and "exploration"(P12). On the 3rd question "what didn't you like about the game?" they answered: "the hot weather" (P5, P13), "the wet terrain" (P1) and one player answered: "I would prefer the linear game structure, with one clue to lead you to the other"(P12). Next, regarding the organization of the game, they were asked to remark or make suggestions for improvements about the game. One player suggested that the search area should be more clearly defined, using, for example, barrier tape to mark its boundaries, because, during the play, the player is absorbed in the game and can easily get out of the area (P12). Another player suggested that the search area could be expanded to include more monuments, allow more player movement, and thus more collaboration (P2).

\subsection{Attitude Toward Local Cultural Heritage}

Three questions related to the content of the AR app and two more general questions were asked to players in order to study possible changes in users' attitude toward the local cultural heritage through the game and the AR app. The first question "What would you like to comment on the content of the application?" aimed to investigate player's opinion 
and to find out what they remember most about the AR app content. About the digital content displayed regarding the monuments of the site, players were impressed about the history of the old church, since they did not know that there was a monastery that was later evolved into a church, while the video of watermill's working days evoked pleasant memories in five of them (P2, P5, P10, P11, P13). About the myth displayed after the riddle solution at the end of the game, they wondered about the representation process. On question "What is your opinion about the representation of the myth?" all players stated that they really liked it, and as they characteristically commented, they liked that they learned about a myth they did not know. Furthermore, they were asked if they consider that this myth's representation is more suitable for a certain age group and specifically only for preschool children. They all answered negatively, since they found it really interesting and believed that this representation successfully conveys the message, i.e., it explains the myth better than other representations such as a black and white video of previous decades or a documentary video (P6).

Then, to the question "how much did you like the combination of culture and technology?" everyone answered "a lot". Also, on question "Would you like similar experiences to be available for this area?" everyone stated "yes, very much". Finally, when asked "how would you describe the overall experience?", they commented: "I liked learning while playing" (P1-P16), "I generally do not like the history but I liked learning the historical facts about my village" (P5), "I was anxious because I thought I should know information in advance and that I would be asked about it" (P8), "it was worth a lot" (P9-P16), "a unique experience" (P9-P16), "I felt like a young child" (P15), "you learn through play" (P1-P8), "very good", "interesting", "fun", "entertaining" (P1-P16).

\section{Discussion and Conclusions}

The first feedback provided by the current case study is encouraging and allows us to answer positively the research questions, without, of course, the possibility of generalizing the results. More specifically:

- regarding the impact of the Serious Escape Game on team's behavior (research question 1), it is noticed that the game experience had positive effects and offered players multiple and valuable benefits such as new knowledge, teamwork, collaboration, enjoyment, motivation, interest etc., corresponding to learning outcomes and skills enhancement and improvement reported in other surveys $[3,10,20,23,24]$ and therefore confirming their results. Additionally, it was found that the friendly competition between the two teams helped players to immerse in the activity and the given ability to players to decide how to play allowed them to engage with the game in their own way, confirms the results and guidelines of other studies [4,9].

- the positive and immediate response to participation, the positive attitude during the game and the positive comments of the players after the end of the game are in line with the results of other surveys [2] and indicate that this category of games can be accepted to a large extent by the players/visitors (research question 2).

- the players' positive attitude, enthusiasm and interest about the local history and cultural heritage and their determination to overcome difficulties of the natural environment are in line with the findings of other studies [20]. Additionally, their positive behavior and attitude indicate respect both for the game and for the space, in contrast to cases of damages in the room caused by players [4] and therefore this type of game can positively affect players' / visitors' attitudes toward local cultural heritage (research question 3).

Overall, the current study reveals that it is feasible to conduct a Serious Escape Game such as Millsecret in outdoor settings. Furthermore, regarding the organization and conduction of this type of game, it is concluded that:

- the role of the game master is very important and it is more efficient for him to be in the same place with the players as it is proposed in escape room games [4] and to watch and provide immediate help if needed, according to other studies guidelines [9]. 
- it is confirmed that debriefing is a critical element for reflection, and it must be a mandatory component, in order to achieved multiple goals, as it has been highlighted in other studies $[3,4,9]$.

- $\quad$ regarding game difficulty [9], it is better to provide easy games, which every team can complete, in order to avoid negative feelings [4] and negative attitude toward the theme of the game.

- allowing the players to select some options of game rules can enhance players engagement, confirming other studies results and guidelines [4].

- the friendly competition can enhance players engagement, confirming other studies [40].

The current research focused on the investigation of the implementation and the effects of Serious Escape Games in outdoor settings, where the AR application plays a decisive role in the game, but without dominating the user experience and without interfering with the natural environment and monuments. A limitation of the game is the activation of the image-based AR application. Since testing and game conduction took place at the same time period with a difference of a few days, there were no difficulties in the AR app activation. However, in tests performed a month later, in the case of the old church's building image recognition was successful, while in the case of the watermill's building it failed. This fact makes the game not replayable in a different time period. The use of hybrid solutions on recognition, with minimum or no intervention in the physical environment and monuments [41], can be a solution to make the game replayable. Another limitation is the learning outcomes evaluation of the Serious Escape Games; however, it is a point that requires further investigation since according to other research measuring the impact of games is an important but difficult task [10,24], where diverse evaluation models [42] and theories such as Flow's theory have to further analyzed and adopted [43] in order for the diverse characteristics of the different game genres to be examined [42,43]. The results of the research and the experience gained from the design and implementation of the game can be used for future adaptations of this type of game experience and allows us to examine several extensions of the game. These extensions could include different places and monuments of the same area, use of both the interior and exterior of a monument where possible. Other extensions will focus on the combined use of different technologies such as Virtual Reality and Internet of Things and the participation of the players in shaping of the game scenario, always giving emphasis on the players' safety and the monuments' protection.

Author Contributions: Conceptualization, S.T. and G.S.; investigation, S.T. and A.B.; methodology, S.T., G.S. and A.B.; supervision, G.S.; writing—original draft, S.T. and A.B.; writing—review \& editing, S.T., G.S. and A.B. All authors have read and agreed to the published version of the manuscript.

Funding: This research received no external funding.

Institutional Review Board Statement: Not applicable.

Informed Consent Statement: Not applicable.

Data Availability Statement: Data sharing is not applicable to this article.

Conflicts of Interest: The authors declare no conflict of interest.

\section{Appendix A}

\section{List of Questions}

Note: All questions were "open-ended" except for the 5th question, where participants were asked to answer by selecting one of the predefined answers of "not at all", "a little", "moderate", "very" and "extremely" for participants' convenience and time saving purposes.

1. Players age and main occupation (asked to select one of the following: pupil, student, other) 
2. Do you often visit the village?

3. Do you know any historical information about the place? If so, how did you learn about it?

4. Have you ever played escape room games?

5. How interesting did you find the game?" (please select one of the following: "not at all", "a little", "moderate", "very" and "extremely")

6. What did you like about the game?

7. What didn't you like about the game?

8. Do you have any comments or suggestions you would like to make about the game?

9. Have you used any AR application? if so, could you please mention one?

10. What would you like to comment on the content of the AR application?

11. What is your opinion about the representation of the myth?

12. Do you believe that this representation is intended only for young children, that is more suitable for a certain age group and specifically only for preschool children?

13. How much did you like the combination of culture and technology?

14. Would you like similar experiences to be available for this area?

15. How would you describe the overall experience?

\section{References}

1. Ćosović, M.; Brkić, B.R. Game-Based Learning in Museums-Cultural Heritage Applications. Information 2020, 11, 22. [CrossRef]

2. Borrego, C.; Fernández, C.; Blanes, I.; Robles, S. Room escape at class: Escape games activities to facilitate the motivation and learning in computer science. J. Technol. Sci. Educ. 2017, 7, 162-171, ISSN 2013-6374. [CrossRef]

3. Fotaris, P.; Mastoras, T. Escape Rooms for Learning: A Systematic Review. In Proceedings of the 13th International Conference on Game Based Learning, ECGBL; Elbaek, L., Majgaard, G., Valente, A., Khalid, S., Eds.; Academic Conferences and Publishing International Limited: Reading, UK, 2019; pp. 235-243. [CrossRef]

4. Nicholson, S. Peeking Behind the Locked Door: A Survey of Escape Room Facilities. White Paper. 2015. Available online: http:/ / scottnicholson.com/pubs/erfacwhite.pdf (accessed on 20 September 2020).

5. Malegiannaki, I.; Daradoumis, T. Analyzing the educational design, use and effect of spatial games for cultural heritage: A literature review. Comput. Educ. 2017, 108, 1-10, ISSN 0360-1315. [CrossRef]

6. Dimitropoulos, K.; Tsalakanidou, F.; Nikolopoulos, S.; Kompatsiaris, I.; Grammalidis, N.; Manitsaris, S.; Denby, B.; CrevierBuchman, L.; Dupont, S.; Charisis, V.; et al. A Multimodal Approach for the Safeguarding and Transmission of Intangible Cultural Heritage: The Case of i-Treasures. IEEE Intell. Syst. 2018, 33, 3-16. [CrossRef]

7. Hautaniemi, A. Combining Games and Cultural Heritage Gamified Experiences Can Help Youth Engagement in Joint History. In Smart Business Annual Review; Sallinen, N., Kotonen, U., Eds.; Painopaikka: Hämeenlinna, Finland, 2019; pp. 56-62. ISBN 978-951-827-316-8.

8. Yang, Y.; Zhang, D.; Ji, T.; Li, L.; He, Y. Designing Educational Games Based on Intangible Cultural Heritage for Rural Children: A Case Study on "Logic Huayao". In Advances in Human Factors in Wearable Technologies and Game Design. AHFE 2018. Advances in Intelligent Systems and Computing; Ahram, T., Ed.; Springer: Cham, Switzerland, 2018; Volume 795. [CrossRef]

9. Guigon, G.; Humeau, J.; Vermeulen, M. A Model to Design Learning Escape Games: SEGAM. In Proceedings of the 10th International Conference on Computer Supported Education (CSEDU 2018); SCITEPRESS: Funchal, Madeira, Portugal, 2018; Volume 2, pp. $191-197$. ISBN 978-989-758-291-2.

10. Warmelink, H.; Mayer, I.; Weber, J.; Heijligers, B.; Haggis, M.; Peters, E.; Max Louwerse, M. AMELIO: Evaluating the Teambuilding Potential of a Mixed Reality Escape Room Game. In Extended Abstracts Publication of the Annual Symposium on ComputerHuman Interaction in Play; ACM: New York, NY, USA, 2017; pp. 111-123. [CrossRef]

11. Antoniou, A. Predicting Cognitive Profiles from a Mini Quiz: A Facebook Game for Cultural Heritage. In Games and Learning Alliance. GALA 2018. Lecture Notes in Computer Science; Gentile, M., Allegra, M., Söbke, H., Eds.; Springer: Cham, Switzerland, 2018; Volume 11385. [CrossRef]

12. Paliokas, I.; Sylaiou, S. A Classification Model for Serious Games Used in Museums, Galleries and Other Cultural Sites. In Strategic Innovative Marketing and Tourism. In Proceedings in Business and Economics; Kavoura, A., Kefallonitis, E., Giovanis, A., Eds.; Springer: Cham, Switzerland, 2019; pp. 1057-1064. [CrossRef]

13. Lu, Z.; Annett, M.; Fan, M.; Wigdor, D. I feel it is my responsibility to stream": Streaming and Engaging with Intangible Cultural Heritage through Livestreaming. In Proceedings of the 2019 CHI Conference on Human Factors in Computing Systems (CHI '19), Glasgow, Scotland, UK, 4-9 May 2019; Association for Computing Machinery: New York, NY, USA, 2019; pp. 1-14. [CrossRef]

14. Hanes, L.; Stone, R. Applying constrained virtual environments to serious games for heritage. Int. J. Serious Games 2019, 6, 93-116. [CrossRef]

15. Panou, C.; Ragia, L.; Dimelli, D.; Mania, K. An Architecture for Mobile Outdoors Augmented Reality for Cultural Heritage. ISPRS Int. J. Geo-Inf. 2018, 7, 463. [CrossRef] 
16. Vocaturo, E.; Zumpano, E.; Caroprese, L.; Pagliuso, S.M.; Lappano, D. Educational Games for Cultural Heritage. In Proceedings of the 1st International Workshop on Visual Pattern Extraction and Recognition for Cultural Heritage Understanding Co-Located with 15th Italian Research Conference on Digital Libraries (IRCDL 2019) CNR Area in Pisa, Pisa, Italy, 30 January 2019; Volume 2320, pp. 95-106.

17. Di Paola, F.; Inzerillo, L.; Alognaa, Y. A GAMING APPROACH FOR CULTURAL HERITAGE KNOWLEDGE AND DISSEMINATION. In Proceedings of the Int. Arch. Photogramm. Remote Sens. Spatial Inf. Sci., XLII-2/W15, 27th CIPA International Symposium "Documenting the Past for a Better Future", Ávila, Spain, 1-5 September 2019; pp. 421-428. [CrossRef]

18. Koo, S.; Kim, J.; Kim, C.; Kim, J.; Cha, H.S. Development of an Augmented Reality Tour Guide for a Cultural Heritage Site. J. Comput. Cult. Herit 2019, 12, 4. [CrossRef]

19. Polyakova, I.R.; Maglieri, G.; Mirri, S.; Salomoni, P.; Mazzeo, R. Art Scene Investigation: Discovering and supporting cultural heritage conservation through Mobile AR. In Proceedings of the IEEE INFOCOM 2019-IEEE Conference on Computer Communications Workshops (INFOCOM WKSHPS), Paris, France, 29 April-2 May 2019; pp. 584-589. [CrossRef]

20. Koutroumanos, G.; Labropoulos, G. "Salamis": A location augmented reality game for local history. In Proceedings of the 11th Pan-Hellenic and International Conference "ICT in Education", Thessaloniki, Greece, 19-21 October 2018; Demetriadis, S., Dagdilelis, V., Tsiatsos, T., Magnisalis, I., Tzimas, D., Eds.; AUTh-UOM: Thessaloniki, Greece, 2018; pp. 355-362. ISBN 978-618-83186-2-5.

21. Shin, J.; Kim, J.; Woo, W. Narrative design for Rediscovering Daereungwon: A location-based augmented reality game. In Proceedings of the IEEE International Conference on Consumer Electronics (ICCE), Las Vegas, NV, USA, 8-11 January 2017; pp. 384-387. [CrossRef]

22. Haahr, M. Creating Location-Based Augmented-Reality Games for Cultural Heritage. In Serious Games. JCSG 2017. Lecture Notes in Computer Science; Alcañiz, M., Göbel, S., Ma, M., Fradinho Oliveira, M., Baalsrud Hauge, J., Marsh, T., Eds.; Springer: Cham, Switzerland, 2017; Volume 10622, pp. 313-318. [CrossRef]

23. Nofal, E.; Panagiotidou, G.; Reffat, R.M.; Hameeuw, H.; Boschloos, V.; Vande Moere, A. Situated Tangible Gamification of Heritage for Supporting Collaborative Learning of Young Museum Visitors. ACM J. Comput. Cult. Herit. 2020, 13, 3. [CrossRef]

24. Mortara, M.; Catalano, C.E.; Bellotti, F.; Fiucci, G.; Houry-Panchetti, M.; Petridis, P. Learning cultural heritage by serious games. J. Cult. Herit. 2014, 15, 318-325, ISSN 1296-2074. [CrossRef]

25. Mortara, M.; Catalano, C.E. 3D virtual environments as effective learning contexts for cultural heritage. Ital. J. Educ. Technol. 2018, 26, 5-21. [CrossRef]

26. What Are Serious Games? Growth Engineering Ltd. Available online: https://www.growthengineering.co.uk/what-are-seriousgames/ (accessed on 15 July 2020).

27. Isa, W.M.W.; Zin, N.A.M.; Rosdi, F.; Sarim, H.M. Serious Game Design for Terengganu Brassware Craft Heritage. In Proceedings of the IEEE Conference on Graphics and Media (GAME), Pulau Pinang, Malaysia, 19-21 November 2019; pp. 13-17. [CrossRef]

28. Yallihep, M.; Kutlu, B. Mobile serious games: Effects on students' understanding of programming concepts and attitudes towards information technology. Educ. Inf. Technol. 2020, 25, 1237-1254. [CrossRef]

29. Tsita, C.; Satratzemi, M. How serious games in cultural heritage are being evaluated. In Proceedings of the 11th Pan-Hellenic and International Conference "ICT in Education", Thessaloniki, Greece, 19-21 October 2018; Demetriadis, S., Dagdilelis, V., Tsiatsos, T., Magnisalis, I., Tzimas, D., Eds.; AUTh-UOM: Thessaloniki, Greece, 2018; pp. 97-104. ISBN 978-618-83186-2-5.

30. Georgopoulos, A.; Kontogianni, G.; Koutsaftis, C.; Skamantzari, M. Serious Games at the Service of Cultural Heritage and Tourism. In Tourism, Culture and Heritage in a Smart Economy; Katsoni, V., Upadhya, A., Stratigea, A., Eds.; Springer: Cham, Switzerland, 2017. [CrossRef]

31. Konstantakis, M.; Caridakis, G. Adding Culture to UX: UX Research Methodologies and Applications in Cultural Heritage. ACM J. Comput. Cult. Herit. 2020, 13, 4. [CrossRef]

32. Clarke, S.J.; Peel, D.J.; Arnab, S.; Morini, L.; Keegan, H.; Wood, O. EscapED: A Framework for Creating Educational Escape Rooms and Interactive Games to For Higher/Further Education. Int. J. Serious Games 2017, 4. [CrossRef]

33. Georgoulas, S. Platanoussa. Epirotiki Estia 1973, 256-258, 500-510.

34. Tzima, S.; Styliaras, G.; Smyris, G.; Bassounas, A. Digital Representations and Cultural Heritage Interactions Through the “Greek Type" Watermills Case. In Proceedings of the 2018 9th International Conference on Information, Intelligence, Systems and Applications (IISA), Zakynthos, Greece, 23-25 July 2018.

35. Tzima, S.; Styliaras, G.; Zaragas, H.; Bassounas, A. The Effects of an Educational Augmented Reality Application on the Interpretation and Communication of Cultural Assets: The "Greek Type" Watermill's Case. In Proceedings of the EdMedia + Innovate Learning, Amsterdam, The Netherlands, 25 June 2018; Association for the Advancement of Computing in Education (AACE): Waynesville, NC, USA, 2018.

36. Tzima, S.; Styliaras, G.; Bassounas, A.; Tzima, M. Harnessing the Potential of Storytelling and Mobile Technology in Intangible Cultural Heritage: A Case Study in Early Childhood Education in Sustainability. Sustainability 2020, 12, 9416. [CrossRef]

37. Tzima, S.; Tzima, M. Stone and wood. Tzoumerkiotika. Chronika 2020, 21, 75-77.

38. Issari, P.; Pourkos, M. Qualitative Research Methodology; Hellenic Academic Libraries Link: Athens, Greece, 2015; pp. 83, 97, 111-112. Available online: http:/ / hdl.handle.net/11419/5826 (accessed on 20 July 2020).

39. Cohen, L.; Manion, L.; Morrison, K. Research Methods in Education, 6th ed.; Routledge: London, UK, 2007 ; p. 475.

40. Hughes, A. Gamification versus Serious Games. LEARNING TECHNOLOGIES, 7 September 2017. Available online: https: //trainingindustry.com/articles/learning-technologies/gamification-versus-serious-games/ (accessed on 8 May 2019). 
41. Tzima, S.; Styliaras, G.; Bassounas, A. Augmented Reality in Outdoor Settings: Evaluation of a Hybrid Image Recognition Technique. J. Comput. Cult. Herit.. under publication.

42. Fokides, E.; Atsikpasi, P.; Kaimara, P.; Deliyannis, I. Factors influencing the subjective learning effectiveness of serious games. J. Inf. Technol. Educ. Res. 2019, 18, 437-466. [CrossRef]

43. Perttula, A.; Kiili, K.; Lindstedt, A.; Tuomi, P. Flow experience in game based learning-A systematic literature review. Int. J. Serious Games 2017, 74, 57-72. [CrossRef] 